\title{
Self-assembled aggregates in the gravitational field: growth and nematic order
}

\author{
Vladimir A. Baulin* \\ Service des Interfaces et des Matériaux Moléculaires et Macromoléculaires, \\ DRFMC, CEA-Grenoble, 17 rue des Martyrs, 38054 Grenoble, Cedex 9, France
}

\begin{abstract}
The influence of the gravitational field on the reversible process of assembly and disassembly of linear aggregates is focus of this paper. Even the earth gravitational field can affect the equilibrium properties of heavy biological aggregates such as microtubules or actin filaments. The gravity gives rise to the concentration gradient which results in the distribution of aggregates of different lengths with height. Strong enough gravitational field induces the overall growth of the aggregates. The gravitational field facilitates the isotropic to nematic phase transition reflecting in a broader transition region. Coexisting phases have notedly different length distributions and the phase transition represent the interplay between the growth in the isotropic phase and the precipitation into nematic phase. The fields in an ultracentrifuge can only reinforce the effect of gravity, so the present description can be applied to a wider range of systems.
\end{abstract}

PACS numbers: 87.65.+y, 87.16.Ka, 82.35.Pq, 82.30.Nr, 82.70.Uv

Keywords: living polymers, liquid crystals, gravity, microtubules, actin filaments, phase diagram

\section{INTRODUCTION}

Living polymerization has a substantial number of industrial applications in the production of soaps, dyes, organometallic complexes, etc.1.2 Moreover, some biological objects owe their functionality to reversible processes of assembly and disassembly. Particular examples are microtubules, actin and intermediate filaments composing the cytoskeleton of living cells 3.4 .5 They are involved in several vital processes such as transport, chromosomal segregation, $\frac{6}{-}$ growth and division of a cell. In contrast to common synthetic living polymers, biological self-assembled objects have a considerable molecular masses, sufficient to induce a significant concentration gradient in an aqueous suspension of these objects. In turn, the concentration gradient can influence the equilibrium size of the objects and for long rigid aggregates it can even provoke the isotropic - nematic phase transition.

Before we address such phenomena it is useful to get an estimate of the relevance of the effect of gravity for a real system. Let us focus on a particular example of microtubules. The typical length of a microtubule is 10 $\mu \mathrm{m}, 7.8$ the linear density is $1.6 \times 10^{5}(\mathrm{~g} / \mathrm{mol}) / \mathrm{nm}$, so the total mass of a microtubule is $\sim 10^{9} \mathrm{~g} / \mathrm{mol}$. The molecular mass of a tubulin dimer, subunit of a microtubule, is $m_{0} \sim 10^{5} \mathrm{~g} / \mathrm{mol}^{3}, \frac{3}{,}$ thus, one microtubule comprises of $N \sim 10^{4}$ tubulins. The influence of the gravity is significant if the potential energy of the object in the gravitational field is of order or higher the thermal energy, $k T$, i.e. if $(m g h / k T) N \equiv \beta N \gtrsim 1$, where $m=m_{0}-\widetilde{\rho} v$ is the

*e-mail: vbaulin@cea.fr

J. Chem. Phys., 119(5), 2874-2885 (2003). mass of a subunit corrected for buoyancy $\left(\widetilde{\rho} \sim 1 \mathrm{~g} / \mathrm{cm}^{3}\right.$ is the density of water, $v \sim 100 \mathrm{~nm}^{3}$ is the subunit volume, so the effective mass in a solution is $m \sim 10^{4} \mathrm{~g} / \mathrm{mol}$ ). Thus, the gravity is important when $\beta \gtrsim \beta^{*}=1 / N$.

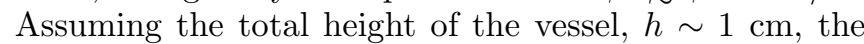
gravitational acceleration, $g \sim 10 \mathrm{~m} / \mathrm{s}^{2}$, the temperature, $T \sim 300 \mathrm{~K}$, we get $\beta \sim 10 \beta^{*}$. So, even the earth gravitational field can influence the equilibrium properties of such heavy objects as microtubules. Note, that one can reach $10^{4}-10^{5}$ times larger gravitational acceleration in an ultracentrifuge,$\frac{9}{,}$ which sometimes is used in the process of preparation of microtubules,$\frac{8}{2}$ or in the pelleting experiments. ${ }^{10.11}$ To this end, the gravitational field can serve as a driving force for the assembly and, eventually, to the nematic ordering of growing aggregates. Another way to study the equilibrium distribution of linear aggregates is the rotating clinostat experiments 12 which constitute the rotation of a tube about the horizontal axis. Changing the direction of the "fall" all the time can effectively reduce the gravity. Such apparatus gives the gravitational fields in the range $10^{-2} \div 10^{2} \mathrm{~g}$.

The rotating clinostat experiments ${ }^{12}$ were used to examine the microtubule self-organization and structuration by reaction-diffusion mechanisms leading to different pattern formation. It was shown, that microtubules selforganize in different patterns in the normal gravitational field, while no self-organization occurs in the absence of the gravity 13.14 .15

It is noteworthy, that the gravity can also bring about the opposite process, namely the floating to the surface when $m_{0}<\widetilde{\rho} v$. One of the most known processes of that type is the creaming of emulsions. In our consideration this only changes the sign of the effective mass, $m$, while the top and the bottom switch their places.

The problem of the influence of weak gravitational fields on different vital processes is largely investigated in the framework of so-called microgravity research performed in sounding rockets, parabolic flights or a space 
station. In particular, it concerns a number of experiments conducted on board of space-shuttle flights. For example, the formation of liposomes is altered by the gravitational field ${ }^{16}$ the size of the liposomes formed in the absence of the gravity is considerably larger than that formed in the normal earth conditions. The gravity also influence the long-range ordering of some systems: ${ }^{17}$ space-grown crystals have better optical quality, smoothness of crystal faces, etc. The problem of crystal growth under gravity is also discussed in ref. 18 .

However, the number of theoretical treatment of these observations is not so impressive. Here are some examples: the size distribution with height of noninteracting linear micelles in the gravitational field has been analyzed in ref. 19. It was shown, that the concentration gradient due to gravitational or centrifugal field leads to the hyperbolic dependence of the average aggregation number with height in contrast to simple barometric dependence for nonaggregating objects. The dynamics of linear micelles in the ultracentrifuge is studied in ref. 20. The sedimentation of colloidal rods in an ultracentrifuge, has been studied theoretically in ref. 21 .

In our previous paper ${ }^{22}$ we studied the isotropic to nematic phase transition of rigid monodisperse rods with fixed size in the gravitational field. The major result is that the gravity can induce the nematic ordering in the system of rigid rods, while the main effect of the gravity on the phase diagram is the broadening of the phase coexistence region. It was also shown that interactions between rigid rods can lead to deviations from the barometric distribution of rods with height.

In the present paper we analyze a wider range of systems. Instead of fixed length of rods ${ }^{22}$ we assume that the size of rods determines by the equilibrium conditions. In particular, this is the case of (i) living polymers, which can attach monomers to the end of the chain, (ii) linear surfactant micelles, (iii) biological self-assembled objects such as microtubules and actin filaments. Although all these systems have different geometries and different size scales, they have additional degree of freedom, namely the annealed length of the aggregates which can be tuned by external conditions. Thus, we expect, that the gravitational field can have a severe influence on the equilibrium size and the concentration profile of the aggregates. The new result of this paper is that the gravity induces the overall growth of the aggregates.

Concerning the isotropic to nematic phase transition of stiff aggregates with annealed size, we expect the same effect of the gravity as in the case of rods with fixed length. Namely, the gravity can induce the nematic ordering and broaden the coexistence region. However, annealed length implies a large polydispersity of the aggregates, thus, we investigate the influence of the gravity on the distribution of lengths in a solution. Since the phase separation is coupled with the redistribution of aggregates of different lengths with different heights, we discuss the structure of the two coexisting phases and the distribution of the average aggregation number with height.

The paper is organized as follows. In the next section the general definitions and the expressions for the free energy and the distribution function are given. In the section III we consider the equilibrium properties of the homogeneous isotropic solution of living polymers under gravity. The concentration profiles and the average aggregation numbers as a functions of height are analyzed. In the section IV, we examine the isotropic to nematic phase transition in the gravitational field. We discuss the effect of the broadness of the phase separation region in the gravitational field for living polymers, the length distribution functions of the aggregates in the coexisting region and polydispersity of the phases. In the Conclusion we summarize the obtained results.

\section{FREE ENERGY AND DISTRIBUTION FUNCTION}

Living polymers are linear aggregates which can bind reversibly one subunit to the end of the chain. They show a broad distribution of lengths, since their energy depends only on end effects and there are no packing constrains preventing the infinite growth as in the case of spherical micelles. The scission end energy (in units of $k T), \delta>0$ ensures energetically favorable formation of aggregates. The aggregation energy per subunit, $\varepsilon_{N}$ has a simple form: ${ }^{23} \varepsilon_{N}=\varepsilon_{\infty}+\delta / N$, where $\varepsilon_{\infty}$ is the bulk energy, which is independent on $N$. Thus, $N \varepsilon_{N}$ is the energy of an aggregate with the aggregation number $N$. The subunit concentration is assumed to be much larger than $C M C$, so the aggregates are rather long.

Our description is based on the theories of living polymers and the liquid crystalline transition in the absence of the field 24.25 .26 .27 .28 .29 .30 .31 .32 .33 .34 For our description we take as a starting point ref. 30. It is known, that the assumption of perfectly rigid aggregates leads to unphysically strong coupling of growth and nematic ordering 30.32 .35

That is why, we have to allow for the partial flexibility of the aggregates. We do it in accordance to refs ${ }^{32.33 .35}$, where the flexibility of the chains is coming through a particular form of the trial function in the nematic phase. However, in this approach the flexibility of the isotropic phase is not taken into account.

We consider the solution of living polymers in the vessel subject to the gravitational field. Suppose, that the gravitational field acts in the $z$ direction, such that the subunit concentration varies from its maximal value at $z=0$ (bottom) to its minimal value at $z=1$ (top). Our discussion is focused on the case of long aggregates. We ignore the end effects, though they can be important for short chains 30 that dominate at the top of the vessel, but for the subunit concentration well above the $C M C$, the fraction of short aggregates in a solution is low.

In the following we will see, that the gravity can lead to the nematic ordering in the described system, thus, 
we write the free energy in a general form which assumes the orientational order. The free energy can be written in terms of the dimensionless concentration of rods each comprised of $N$ subunits oriented in the spatial angle $\Omega$ on the height $z$ as $C(N, \Omega, z)$ (this is the number concentration multiplied by the volume of a single subunit, $v$ ). $C(N, \Omega, z)$ obeys the following constraint

$$
\int N C(N, \Omega, z) d N d \Omega=\phi(z)
$$

where $\phi(z)$ is the total volume fraction of subunits on the height $z$. The total subunit concentration is $\Phi=$ $\int \phi(z) d z$.

The free energy is composed of an ideal and an interaction terms, $F=F_{i d}+F_{\text {int }}$. The ideal term per unit volume is written in the form

$$
\begin{aligned}
\frac{F_{i d}(z)}{k T}= & \int C(N, \Omega, z)[\ln (4 \pi C(N, \Omega, z))-1+ \\
& \left.N \varepsilon_{N}\right] d N d \Omega+\int C(N, \Omega, z) \beta N z d N d \Omega(2)
\end{aligned}
$$

The first term is the well known expression for the free energy of self-assembled aggregates ${ }^{23}$ The factor $4 \pi$ is introduced for convenience of description of the isotropic phase, though it can be absorbed in the energy of an aggregate, $N \varepsilon_{N}$. The last term is the potential energy in units $k T$ of aggregates in the gravitational field. The only parameter associated with the field is $\beta=(m g h) / k T$, which depends on the total height of the vessel. Note, that although the last term in (2) coincide with that in the case of monodisperse rods of fixed length, ${ }^{22}$ the definitions of this parameter does not coincide. Here $m$ is the mass of a single subunit, while in ref. $22 \mathrm{~m}$ is the total mass of a rod. Again, we assume that the characteristic length of the variation of the field is much bigger than the length of a rod, although the fields in the ultracentrifuge can be very high and this assumption can be violated.

In order to apply the Onsager approximation $\stackrel{35,36}{\sqrt{5 e}}$ assume excluded volume interactions only. Moreover, only pair interactions are taken into account, higher order interaction terms are neglected. This is a strong approximation which is strictly speaking not valid for high concentrations in the nematic phase. As we will see, the strong gravitational field induces the precipitation of extremely long, and thus, heavy, aggregates and the second virial approximation should be corrected.

Nevertheless, this approximation works well in the isotropic phase and it also gives reasonable qualitative estimates in the nematic phase.

The interaction term per unit volume for athermal aggregates, written in the second virial approximation has the following form ${ }^{30.33}$

$$
\frac{F_{\text {int }}(z)}{k T}=\frac{1}{v} \int C(N, \Omega, z) C\left(N^{\prime}, \Omega^{\prime}, z\right) B_{2}(\gamma) d N d N^{\prime} d \Omega d \Omega^{\prime}
$$

where $B_{2}(\gamma)$ is the second virial coefficient which depends on the angle $\gamma$ between two rods. Neglecting the end effects, it can be written in the form ${ }^{35.36} B_{2}(\gamma)=$ $L L^{\prime} D|\sin \gamma|$, where $L$ and $L^{\prime}$ are the lengths of two cylinders, $D$ is their diameter. We can rewrite this expression in terms of aggregation numbers $N, N^{\prime}$ and the volume of a subunit, $v$, as $B_{2}(\gamma)=\varkappa v N N^{\prime}|\sin \gamma|$, where $\varkappa$ is the parameter associated with the form of a subunit. In the case of cubic subunits, $\varkappa=1$, while for cylindrical subunits, $\varkappa=4 l / D$, where $l$ is the height of a cylinder. However, to apply this notation to the case of microtubules, we should note, that a microtubule has complex geometry. Microtubules are hollow cylinders consisting of $f=13$ columns of tubulin on the surface ${ }^{3}$ So, they grow up at $f$ places at the same time. Thus, the length of a microtubule $L \sim f N$ and $\varkappa=4 l f^{2} / D$. In the following we assume $\varkappa=1$ for simplicity.

Variation of the free energy (21), (3) with respect to the concentration $C(N, \Omega, z)$ along with the constraint condition (II) leads to the following expression

$$
\begin{aligned}
C(N, \Omega, z)= & \frac{1}{4 \pi} \exp \left(N\left(\mu-\varepsilon_{N}-\beta z\right)-\right. \\
& \left.\frac{2}{v} \int C(N, \Omega, z) B_{2}(\gamma) d N d \Omega\right)
\end{aligned}
$$

where $\mu$ is the Lagrange multiplier, which can be eliminated with the help of the constraint (11).

Introducing the average aggregation number of rods in the direction $\Omega$ at height $z$ as

$$
\bar{N}(\Omega, z)=\frac{\int N C(N, \Omega, z) d N}{\int C(N, \Omega, z) d N}
$$

we can write the expression for the distribution function $C(N, \Omega, z)$ in a simpler form

$$
\begin{aligned}
C(N, \Omega, z) & =\frac{1}{4 \pi} \exp \left(-\frac{N}{\bar{N}(\Omega, z)}-\delta\right) \\
& =\frac{\phi}{\int \bar{N}^{2}(\Omega, z) d \Omega} \exp \left(-\frac{N}{\bar{N}(\Omega, z)}\right)
\end{aligned}
$$

To write the last equality we used the constraint condition (11).

The final expressions could be considerably simplified if we note, that (i) there is no angular dependence in the isotropic phase, (ii) in the nematic phase we can split the distribution function $C(N, \Omega, z)$ in two parts, 28 one dependent on the aggregation number, $N$, and another dependent on spatial angles, $\Omega$. Namely, we denote the length distribution function, $P(N, z)=\int N C(N, \Omega, z) d \Omega$ with the normalization condition $\int P(N, z) d N=\phi(z)$ and the orientation distribution function $R(N, \Omega, z)=\frac{N C(N, \Omega, z)}{\int N C(N, \Omega, z) d \Omega}$ with the normalization $\int R(N, \Omega, z) d \Omega=1$. With this, $C(N, \Omega, z)=$ 
$\frac{P(N, z)}{N} R(N, \Omega, z)$ and the free energy, $F=F_{i d}+F_{\text {int }}$, now yields in the form

$$
\begin{aligned}
& \frac{F(z)}{k T}=\int \frac{P(N, z)}{N} d N\left[\sigma(N, z)+\ln \frac{P(N, z)}{N}-1+\right. \\
& \left.N \varepsilon_{N}+\beta N z+\frac{\pi}{4} N \int P\left(N^{\prime}, z\right) \rho\left(N, N^{\prime}, z\right) d N^{\prime}\right]
\end{aligned}
$$

where $\sigma(N, z)=\int R(N, \Omega, z) \ln 4 \pi R(N, \Omega, z) d \Omega$ is the orientational entropy, $\rho\left(N, N^{\prime}, z\right)=\frac{4}{\pi} \int R(N, \Omega, z)$ $R\left(N^{\prime}, \Omega^{\prime}, z\right)|\sin \gamma| d \Omega d \Omega^{\prime}$ is the excluded volume term. We keep this nomenclature in order to be consistent with refs ${ }^{22.30,32.33 .35 .36}$ In the isotropic phase $\sigma_{i}=0, \rho_{i}=1$ and $R_{i}(N, \Omega, z)=1 / 4 \pi$, so the expression for the free energy simplifies significantly.

In the next section we describe the concentration profile and the average length of the aggregates assuming that the solution of rods is homogeneous and isotropic for the whole range of $z$.

\section{HOMOGENEOUS SOLUTION: GROWTH OF AGGREGATES}

Since the directions of rods in the isotropic phase are completely disordered, the distribution function does not depend on angles, $C_{i}(N, \Omega)=C_{i}(N)$ and eqs. (4), (5) give ${ }^{37}$ the well known result for the average aggregation number in the isotropic phase $\bar{N}_{i}(z)=\sqrt{\phi_{i}(z) e^{\delta}}$. In turn, the free energy expressed in terms of the length distribution function, $P(N, z)$ (17) leads to the free energy of the isotropic phase in the form

$$
\frac{F_{i}(z)}{k T}=\phi_{i}(z)\left[\varepsilon_{\infty}+\beta z-\frac{2}{\bar{N}_{i}(z)}+\frac{\pi}{4} \phi_{i}(z)\right]
$$

Here we used again the phenomenological expression for the aggregation energy (per $k T$ ) of linear aggregates, 23 $\varepsilon_{N}=\varepsilon_{\infty}+\delta / N$. The chemical potential $\mu_{i}(z)=$ $\partial F_{i}(z) / \partial \phi_{i}(z)$ is

$$
\frac{\mu_{i}(z)}{k T}=\varepsilon_{\infty}+\beta z-\frac{1}{\bar{N}_{i}(z)}+\frac{\pi}{2} \phi_{i}(z)
$$

and the osmotic pressure $p_{i}(z)=1 / v \phi_{i}^{2} \partial\left[F_{i} / \phi_{i}\right] / \partial \phi_{i}(z)$ is

$$
\frac{p_{i}(z)}{k T} v=\phi_{i}(z)\left[\frac{1}{\bar{N}_{i}(z)}+\frac{\pi}{4} \phi_{i}(z)\right]
$$

Note, that these expressions formally have the same form as in the absence of gravity ${ }^{30.33}$ (the only difference is that the concentration $\phi_{i}$ depends on $z$ ). This is because on the lengths smaller than the variation of gravitational field, the system does not "feel" the field, since

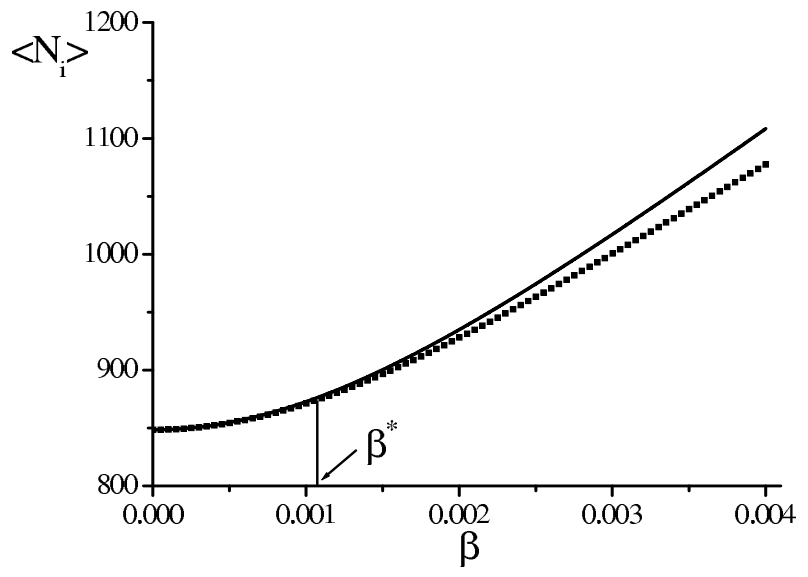

FIG. 1: Average aggregation number on the whole range of heights, $\left\langle N_{i}\right\rangle$, of ideal linear aggregates (14) as a function of a dimensionless parameter associated with the gravitational field, $\beta=m g h / k T$ (solid line) in comparison with the exact solution for nonideal aggregates (squares). The parameters used: $\delta=25, \Phi=10^{-5}$ (low concentration limit). The crossover between the week field and the strong field, which induce considerable growth of aggregates is $\beta^{*} \sim 1 / \bar{N}_{i, 0}$.

all aggregates have almost the same potential energy. In our approximation the gravitational field influence the system only through the concentration gradient. The expressions (9), (10) will be of use to the phase coexistence.

In the equilibrium the chemical potential does not depend on height. Thus, $\mu_{i}(z)=$ const, eq. (9), determines the concentration profile induced by the field. In turn, the variation of the concentration with height leads to a redistribution of aggregates with different lengths at different heights. To address this issue, we focus on two limits: ideal aggregates (low concentrations) and considerably long aggregates (high concentrations).

In the limit of noninteracting ideal aggregates in the gravitational field, 19 the last term in the expression for chemical potential (91) arising from the excluded volume interactions is eliminated. This leads to the following concentration profile

$$
\phi_{i}(z)=\frac{\phi_{i, 0}}{\left(1+\sqrt{\phi_{i, 0} e^{\delta}} \beta z\right)^{2}}
$$

where $\phi_{i, 0}=\phi_{i}(z=0)$ is the concentration at the bottom. This expression is interesting because it shows nonbarometric dependence as expected for the case of nonaggregating objects ${ }^{22}$

The average aggregation number, $\bar{N}_{i}(z)=\sqrt{\phi_{i}(z) e^{\delta}}$, has the hyperbolic dependence on the height $z .19$ Denoting the average aggregation number at the bottom, $\bar{N}_{i, 0}=\bar{N}_{i}(z=0)$, it yields

$$
\bar{N}_{i}(z)=\frac{\bar{N}_{i, 0}}{1+\bar{N}_{i, 0} \beta z}
$$




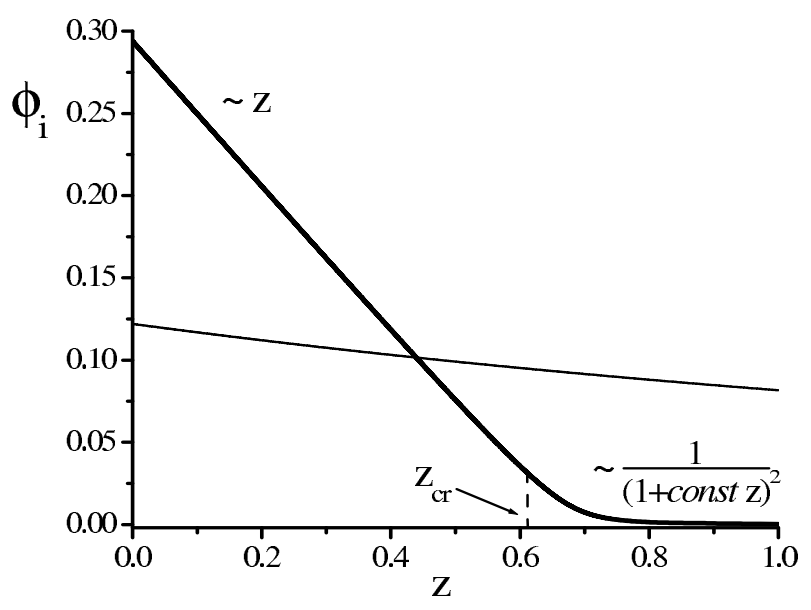

FIG. 2: Typical concentration profile of living polymers subject to the gravitational field in a dilute solution (thick line) for $\beta=0.7, \delta=11$ and the total concentration $\Phi_{i}=0.1$. The dependence $\phi_{i}(z)$ is linear except the region on the top where the concentration is small. The crossover between the regimes is $z_{c r}=\sqrt{\Phi_{i} \pi / \beta}$. In contrast, the concentration profile of nonaggregating subunits $(\delta=0)$ for the same total concentration and the field (thin line) obeys the barometric distribution.

This expression shows, that the aggregates at the bottom are longer than that on the top. This is simply a consequence of the redistribution of subunits with height. It does not tell about the change of the equilibrium size of the aggregates. In order to examine the effect of gravity on the system as a whole, one can consider the global average on the whole range of heights, $\left\langle N_{i}\right\rangle$, as a function of $\beta=(m g h) / k T$. This average is related to the total subunit concentration, $\Phi_{i}=\int \phi_{i}(z) d z$, which assumes to be a constant

$$
\left\langle N_{i}\right\rangle=\frac{\int N C_{i}(N, z) d N d z}{\int C_{i}(N, z) d N d z}=\frac{\Phi_{i} e^{\delta}}{\int \bar{N}_{i}(z) d z}
$$

If the influence of the gravitational field is rather weak, i.e. when $\beta \ll \beta^{*} \sim 1 / \bar{N}_{i, 0}$, it coincides with the expression in the absence of the field, $\left\langle N_{i}\right\rangle \sim \sqrt{\Phi_{i} e^{\delta}}$. Thus, low gravitational field induces only the redistribution of aggregates with height and it does not influence the micellar equilibrium properties. On the other hand, if $\beta \gtrsim \beta^{*}$, $\left\langle N_{i}\right\rangle$ yields 38

$$
\left\langle N_{i}\right\rangle=\frac{\Phi_{i} e^{\delta} \beta}{\ln \left[1+\frac{1}{2} \Phi_{i} e^{\delta} \beta^{2}\left(1+\sqrt{1+\frac{4}{\Phi_{i} e^{\delta} \beta^{2}}}\right)\right]}
$$

This is an increasing function of $\beta$, i.e. strong enough gravitational field is able to induce considerable aggregation even of ideal micelles. The typical plot of this function is presented in Fig. 1. Note, that this expression is valid only for extremely low concentrations, $\Phi_{i} \ll 1$.

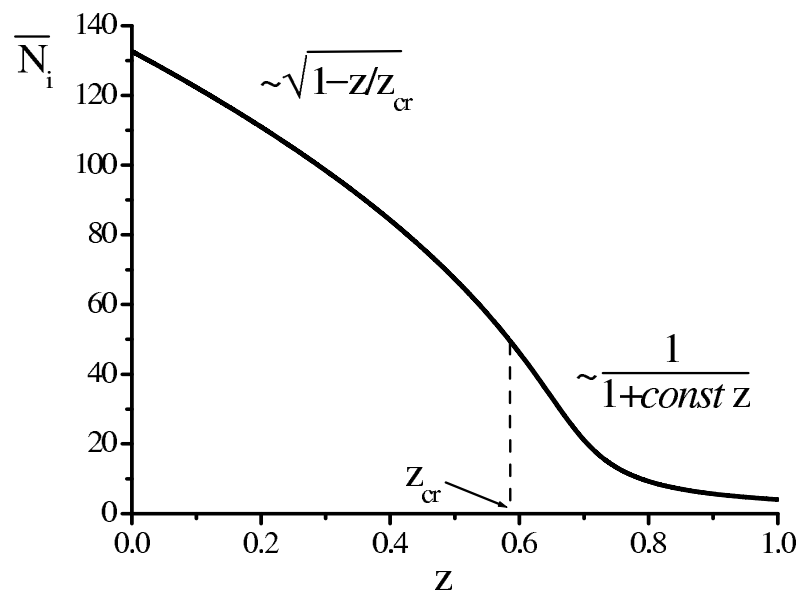

FIG. 3: The average aggregation number of living polymers in a dilute solution, $\bar{N}_{i}(z)$, as a function of the height $z$ for the same parameters as in Fig. 2. The plot can be approximated by two asymptotes: long aggregates, interacting via the second virial approximation for $z<z_{c r}$, and ideal aggregates for $z>z_{c r}$ with $z_{c r}=\sqrt{\Phi_{i} \pi / \beta}$.

However, the deviation of this expression from the exact solution (with the interaction term) is rather small. We can also calculate the asymptote for $\beta \ll 1$,

$$
\left\langle N_{i}\right\rangle \approx \sqrt{\Phi_{i} e^{\delta}}+\frac{1}{24}\left(\Phi_{i} e^{\delta}\right)^{3 / 2} \beta^{2}+O\left(\beta^{4}\right)
$$

If the concentration is not low, we can still get the analytical result in the limit of long aggregates, i.e. when we can neglect the third term in eq. (9). This can be done for high enough end energies $\delta$, i.e. in the formal limit, $\delta \gg 1$. In this case, the concentration depends linearly on the height,

$$
\phi_{i}(z)=\phi_{i, 0}-\frac{2}{\pi} \beta z
$$

while the average aggregation number yields in the form

$$
\bar{N}_{i}(z)=\sqrt{\bar{N}_{i, 0}^{2}-\frac{2}{\pi} e^{\delta} \beta z}
$$

where $\bar{N}_{i, 0}=\sqrt{e^{\delta}\left(\Phi_{i}+\beta / \pi\right)}$ is its value at the bottom, $z=0$. This expression brings us immediately to the conclusion, that the interactions between aggregates change the functional form of $\phi_{i}(z)$ and $\bar{N}_{i}(z)$. In the limit of long aggregates $\phi_{i}(z)$ is a linearly decreasing function and $\bar{N}_{i}(z)$ varies as a square root of height. Although the average length of the aggregates can be hardly measured, the concentration profile is easily accessible experimentally and the dependence of $\phi_{i}(z)$ can be tested directly. The integration of eq. (13) gives the expression for the global average $\left\langle N_{i}\right\rangle$ in the range of all heights in the form 


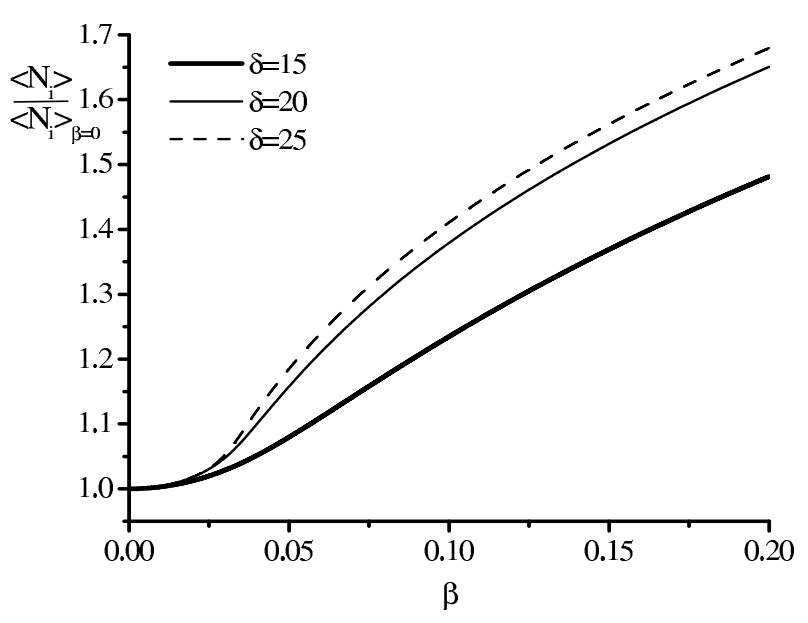

FIG. 4: Numerical plot of the global average aggregation number on the whole range of heights, $\left\langle N_{i}\right\rangle$ of nonideal linear aggregates, rescaled to its value in the absence of the field, $\left\langle N_{i}\right\rangle_{\beta=0}$, as a function of $\beta=m g h / k T$ for different end energies: $\delta=15$ (thick line, $\bar{N}_{i, 0} \approx 200$ ), $\delta=25$ (thin line, $\bar{N}_{i, 0} \approx 2000$ ), $\delta=25$ (dash line, $\bar{N}_{i, 0} \approx 25000$ ). The curve was calculated by the integration of eq. (13) for $\Phi_{i}=0.01$ (high concentration).

$$
\left\langle N_{i}\right\rangle=\frac{\Phi_{i} e^{\delta} \beta}{\frac{\pi}{3} e^{\delta / 2}\left[\left(\Phi_{i}+\frac{\beta}{\pi}\right)^{3 / 2}-\left(\Phi_{i}-\frac{\beta}{\pi}\right)^{3 / 2}\right]}
$$

Similarly to the case of ideal aggregates, $\left\langle N_{i}\right\rangle \sim \sqrt{\Phi_{i} e^{\delta}}$ for $\beta \ll \beta^{*} \sim 1 / \bar{N}_{i, 0}$, while for strong fields it grows up with $\beta$, indicating the increasing number of long aggregates:

$$
\left\langle N_{i}\right\rangle=\sqrt{\Phi_{i} e^{\delta}}+\frac{1}{24} \frac{\sqrt{\Phi_{i} e^{\delta}}}{\Phi_{i} \pi^{2}} \beta^{2}+O\left(\beta^{4}\right)
$$

It is noteworthy, that the increasing $\beta$ induces not only the growth of the aggregates, it also leads to the precipitation of the aggregates to the bottom. At some point, the subunit concentration on the top will be close to zero and the corresponding average aggregation numbers will not be high enough to hold this approximation. To this end, the resulting curve of the concentration profile, $\phi_{i}(z)$ and the average $\bar{N}_{i}(z)$ could be viewed as a combination of the above two limits. Namely, $\bar{N}_{i}(z) \sim \sqrt{1-z / z_{c r}}$ at the bottom $\left(0<z<z_{c r}\right)$ and $\bar{N}_{i}(z) \sim(1+$ const $z)^{-1}$ on the top $\left(z_{c r}<z<1\right)$. The crossover between the regimes, $z_{c r}$, can be roughly considered as a brake up of the approximation of long aggregates (17), $\bar{N}_{i}\left(z=z_{c r}\right)=0$. That gives ${ }^{39}, z_{c r}=\sqrt{\Phi_{i} \pi / \beta}$. The concentration profile is presented in Fig. 2 and the average length corresponding to that profile depicted in Fig. 3. Due to self-assembly, the influence of the gravitational field on the concentration profile of living polymers is

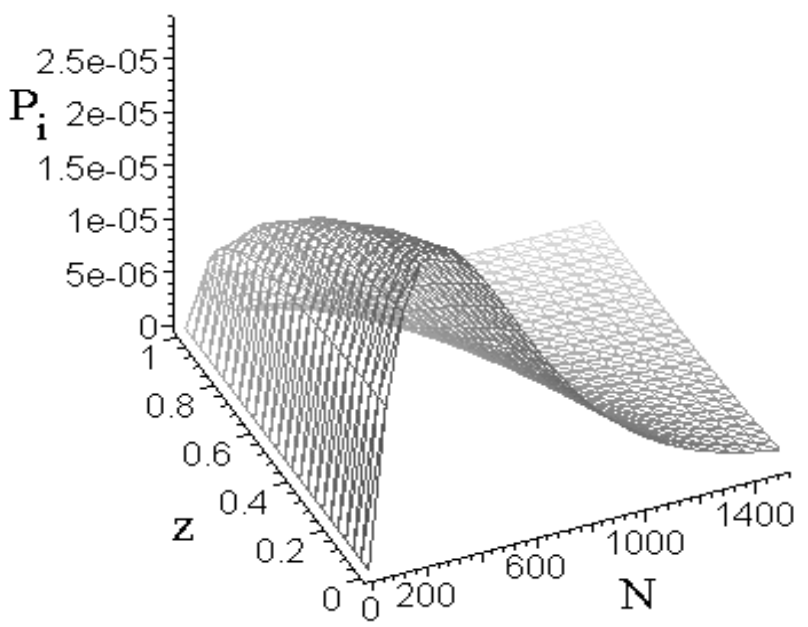

FIG. 5: Three dimensional plot of the distribution function of the aggregate lengths at different heights in the isotropic phase $P_{i}(N, z)$ (20) for $\delta=15, \beta=0.03, \Phi_{i}=0.01$. All the cross-sections with constant heights have the same functional form.

much stronger than that for nonaggregating particles. This is an important conclusion since concentration profiles can be measured directly.

The plot of $\left\langle N_{i}\right\rangle$ for the whole range of the fields $\beta$ can be obtained numerically only. Eqs. $\mu_{i}(z)=$ const (9) and the normalization condition $\Phi_{i}=\int_{0}^{1} \phi_{i}(z) d z$ gives the concentration profile as a function of the total subunit concentration $\Phi_{i}$. The profile can be converted into the average length as $\bar{N}_{i}(z)=\sqrt{\phi_{i}(z) e^{\delta}}$ and inserted into eq. (13). The result is presented in Fig. 4. We plot the ratio $\left\langle N_{i}\right\rangle /\left\langle N_{i}\right\rangle_{\beta=0}$, where $\left\langle N_{i}\right\rangle_{\beta=0}$ is its value in the zero field. This allows us to compare the relative growth of the aggregates with different end energies, $\delta$. As we see, the aggregates growth significantly in the gravitational field, especially for large $\delta$.

At this point it is important to address the question of the magnitude of the gravitational effect for a real system. The typical example of microtubules used in the Introduction gives the value of $\beta$ of the order of 0.001 , which is significantly lower than the values in Fig. 4. These values can correspond to rotating clinostat experiments 12 performed on microtubules samples, where the system rotates about the horizontal axis. The gravitational field in these experiments can be up to $300 \mathrm{~g}$. Moreover, the influence of the gravity on the global average $\left\langle N_{i}\right\rangle$ is noticeable even for normal gravitational conditions in systems with low subunit concentrations: taking $\Phi_{i}=10^{-5}$ and $\delta=30$ we get the average in the absence of the gravity $\left\langle N_{i}\right\rangle_{\beta=0}$ of the order of 10000 , while for $\beta=0.001$ it yields $\left\langle N_{i}\right\rangle \sim 16000$, i.e. the normal gravitational field induces considerable (60\%) growth of the aggregates.

However, $\left\langle N_{i}\right\rangle$ cannot show the redistribution of aggregates of different lengths with height. In order to illustrate the redistribution of aggregates in the gravitational 
field, it is useful to consider the length distribution function $P_{i}(N, z)=\int N C_{i}(N, z) d \Omega=4 \pi N C_{i}(N, z)$. The eq. (6) gives

$$
P_{i}(N, z)=N \exp \left(-\frac{N}{\bar{N}_{i}(z)}-\delta\right)
$$

where we assume $\bar{N}_{i}(z)$ in the form (17). The three dimensional plot of this function is given in Fig. 5 . It is noteworthy, that the envelope curve has the square root dependence in accordance with (17). The total number of aggregates, i.e. the integral of $P_{i}(N, z)$ on $N$, is much higher at the bottom than on the top. The maximum of the curve shifts to higher values of $N$ at the bottom of the vessel.

\section{NEMATIC ORDERING AND PHASE EQUILIBRIUM IN THE GRAVITATIONAL FIELD}

Once the subunit concentration exceeds some critical value, the nematic phase appears at the bottom of the vessel and the system splits into two coexisting phases. It is known, that the average length of the aggregates in the absence of gravity is different in the nematic and the isotropic phases. ${ }^{28,30}$ In this chapter we focus on the following questions: (i) how the gravitational field influence the average length of both phases? (ii) is the growth in the nematic phase as strong as in the isotropic phase? (iii) what would be the distribution function on lengths of the aggregates in the isotropic and the nematic phases as well as the change of the overall distribution function of both phases with increasing gravity? (iv) In particular, we will try to answer the question: what is more favorable for an aggregate: to grow up in the isotropic phase until some critical length and then to change to the nematic phase, or to drop out into nematic phase immediately in order to decrease its potential energy in the field? In any case, it is quite obvious, that the aggregates in the isotropic phase will shorten, while the aggregates in the nematic phase will grow up with increasing gravity.

The simplest way to treat the nematic phase is to employ the variational method $\operatorname{mos}^{35,36}$ that assumes the trial orientational distribution function with the adjustable variational parameter $\alpha$, which is found from the minimization of the free energy. In analogy to the case in the absence of gravity, ${ }^{33,34,35}$ we may write the interaction function $\rho_{n}(z)=4 / \sqrt{\pi \alpha(z)}$ and the entropy function $\sigma_{n}(z)=\ln (\alpha(z) / 4)+N \alpha(z) /(4 p)$, where $p$ is the persistence length of the rod per the length of one subunit 35 The last term reflects the flexibility of the rods. The only difference with the expression in the absence of the field ${ }^{33}$ is that $\alpha$ now depends on the height. Insertion of the expressions for $\sigma_{n}(z)$ and $\rho_{n}(z)$ into (7) and the minimization with respect to $P(N, z)$ along with the constraint $\int P_{n}(N, z) d N=\phi_{n}(z)$ gives the expression for the distribution function $P_{n}(N, z)$ in the form

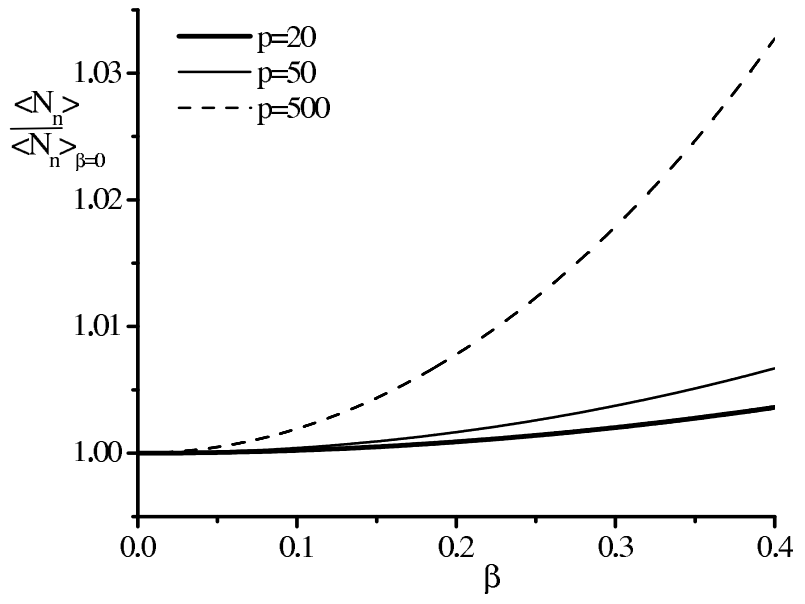

FIG. 6: The global average aggregation number on the whole range of heights in the nematic phase, $\left\langle N_{n}\right\rangle$, eq. (30), rescaled to its value in the absence of the field, $\left\langle N_{n}\right\rangle_{\beta=0}$, as a function of $\beta=m g h / k T$ with different flexibility of the aggregates: $p=20$ (thick line, $\left.\bar{N}_{n, 0} \approx 800\right), p=50$ (thin line, $\bar{N}_{n, 0} \approx$ 600 ), $p=500$ (dash line, $\bar{N}_{n, 0} \approx 300$ ), for $\delta=15, \Phi_{n}=0.6$. The overall growth is negligible with respect to the isotropic phase, Fig. 4.

$$
\begin{aligned}
& \frac{P_{n}(N, z)}{N}=\exp \left(N \left[\mu-\varepsilon_{\infty}-\beta z-\right.\right. \\
& \left.\left.\frac{\pi}{2} \int P_{n}\left(N^{\prime}, z\right) \rho_{n}(z) d N^{\prime}\right]-\sigma_{n}(z)-\delta\right)
\end{aligned}
$$

or applying the constraint (1) we can rewrite

$$
\frac{P_{n}(N, z)}{N}=\frac{\phi_{n}}{\bar{N}_{n}^{2}(z)} \exp \left(-\frac{N}{\bar{N}_{n}(z)}\right)
$$

where the average aggregation number in the nematic phase yields

$$
\bar{N}_{n}(z)=\sqrt{\frac{\alpha(z)}{4} \phi_{n}(z) e^{\delta}}
$$

With this, the free energy of the nematic phase is

$$
\begin{aligned}
\frac{F_{n}(z)}{k T}= & \phi_{n}(z)\left[\varepsilon_{\infty}+\beta z+\frac{\alpha(z)}{4 p}-\frac{2}{\bar{N}_{n}(z)}+\right. \\
& \left.\frac{\pi}{4} \phi_{n}(z) \frac{4}{\sqrt{\pi \alpha(z)}}\right]
\end{aligned}
$$

Minimization of the free energy with respect to $\alpha(z)$ in the limit of considerably long rods, $\bar{N}_{n}(z) \gg 1$, allows to relate $\alpha(z)$ and the local concentration as

$$
\alpha(z)=\left(2 \sqrt{\pi} p \phi_{n}(z)\right)^{2 / 3}
$$




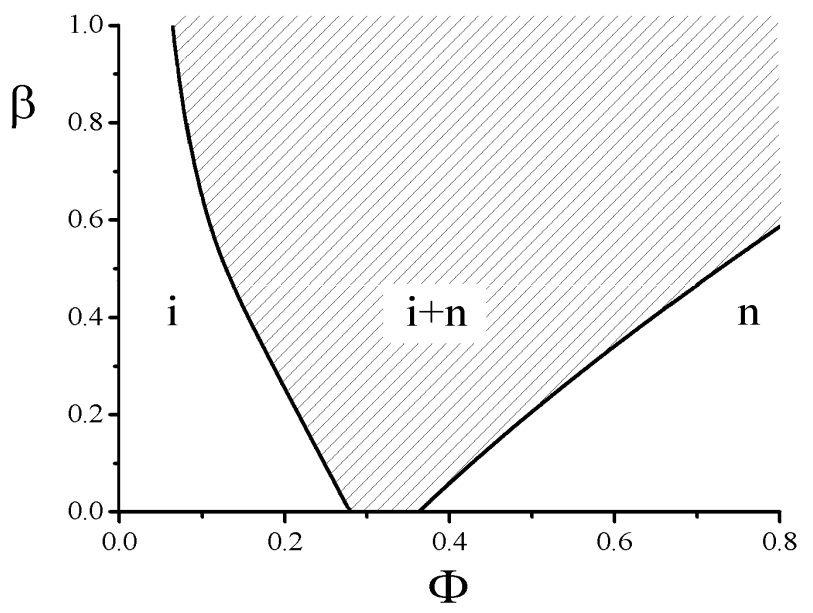

FIG. 7: Phase diagram of living polymers in the gravitational field in the variables: the total subunit concentration $\Phi$ and the parameter associated with the gravitational field $\beta=m g h / k T$ for the parameters $p=40, \delta=10$ leading to the phase transition even in the absence of gravity. The concentrations corresponding to the onset of the phase separation diverge and the coexistence region becomes broader. Here $\mathrm{i}$ denotes the isotropic phase, $\mathrm{n}$ is the nematic phase, $\mathrm{i}+\mathrm{n}$ corresponds to the phase coexistence region.

The chemical potential $\mu_{n}(z)=\partial F_{n}(z) / \partial \phi_{n}(z)$ is

$$
\frac{\mu_{n}(z)}{k T}=\varepsilon_{\infty}+\beta z+\frac{\alpha(z)}{4 p}-\frac{1}{\bar{N}_{n}(z)}+\frac{\pi}{2} \phi_{n}(z) \rho_{n}(z)
$$

and the osmotic pressure $p_{n}(z)=\phi_{n}^{2} / v \partial\left[F_{n} / \phi_{n}\right] / \partial \phi_{n}(z)$ is

$$
\frac{p_{n}(z)}{k T} v=\phi_{n}(z)\left[\frac{1}{\bar{N}_{n}(z)}+\frac{\pi}{4} \phi_{n}(z) \rho_{n}(z)\right]
$$

The form of these expressions is close to that in the absence of gravity $\underline{\underline{33}}$

Let us first calculate the average aggregation number, $\bar{N}_{n}(z)$, in the limit of long aggregates, i.e. when $\delta$ is large, so we can neglect the fourth term in (26). Noting that $\frac{\pi}{2} \phi_{n}(z) \rho_{n}(z)=\alpha(z) / p$, the chemical potential (26) is

$$
\frac{\mu_{n}(z)}{k T} \approx \varepsilon_{\infty}+\beta z+\frac{5}{4} \frac{\alpha(z)}{p}
$$

Thus, $\alpha(z)$ changes linearly with height, $\alpha(z)=\alpha_{n, 0}-$ $4 / 5 p \beta z$, where $\alpha_{n, 0}=\alpha(z=0)$. The local concentration, is then $\phi_{n}(z)=\left(\phi_{n, 0}^{2 / 3}-4 / 5 p^{1 / 3} \beta z /(2 \sqrt{\pi})^{2 / 3}\right)^{3 / 2}$ with $\phi_{n, 0}$ being the concentration at the bottom, and correspondingly, the average aggregation number is

$$
\bar{N}_{n}(z)=\left[\bar{N}_{n, 0}^{4 / 5}-\frac{4}{5}\left(\frac{e^{\delta}}{8 \sqrt{\pi} p}\right)^{2 / 5} p \beta z\right]^{5 / 4}
$$

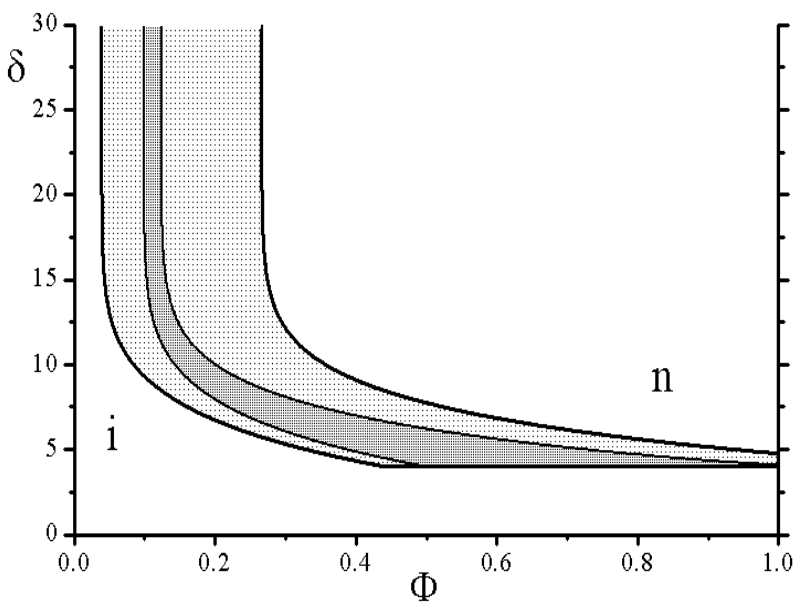

FIG. 8: Phase diagram of living polymers in the gravitational field in the variables: the total subunit concentration $\Phi$ and the end energy of linear aggregates $\delta$ for $p=100$. The dark pattern corresponds to the phase separation region in the absence of the gravity, $\beta=0$, while the light pattern corresponds to the gravitational field $\beta=0.2$, i denotes the isotropic phase, $n$ denotes the nematic phase. The phase separation region becomes broader in the gravitational field.

where $\bar{N}_{n, 0} \equiv \bar{N}_{n}(z=0)=\frac{1}{2} \sqrt{\alpha_{n, 0} \phi_{n, 0} e^{\delta}}$. Hence, we can conclude that $\bar{N}_{n}(z)$ changes almost linearly with height in contrast to the square root behavior in the isotropic phase. Since the nematic phase is composed mostly of long aggregates, the approximation of long aggregates works well in a large range of concentrations.

Assuming, that the vessel is entirely occupied by the nematic phase, we can calculate the global average length of the aggregates on the whole range of heights

$$
\left\langle N_{n}\right\rangle=\frac{\Phi_{n} e^{\delta}}{\int \bar{N}_{n}(z) d z}
$$

Although an analytical expression for this case is not possible, we can plot the numerical result. Integrating eq. (29) and fixing the total concentration $\Phi_{n}=\int_{0}^{1} \phi_{n}(z) d z$, we get with the help of eqs. (23) and (25) the dependence of $\left\langle N_{n}\right\rangle$ on $\beta$ (Fig. 6). Here again we plot it as a rescaled variable, $\left\langle N_{n}\right\rangle /\left\langle N_{n}\right\rangle_{\beta=0}$, in order to compare the growth of aggregates with different stiffness, $p$. As in the case of the isotropic phase, the gravitational field leads to the growth of the aggregates, though the growth in the nematic phase is less pronounced. One can see that stiff aggregates ( $p$ is large) growth better than flexible ones ( $p$ is small). By further increasing $\beta$ the top of the vessel rarefies until the appearance of the isotropic phase coexisting with the nematic phase at the bottom.

To treat the phase separation region we can employ the same method as in ref. 22 which is valid for the coexisting phases with concentration gradients. Once a system is subject to a concentration gradient, there is no concentration which determines the equilibrium properties of a 


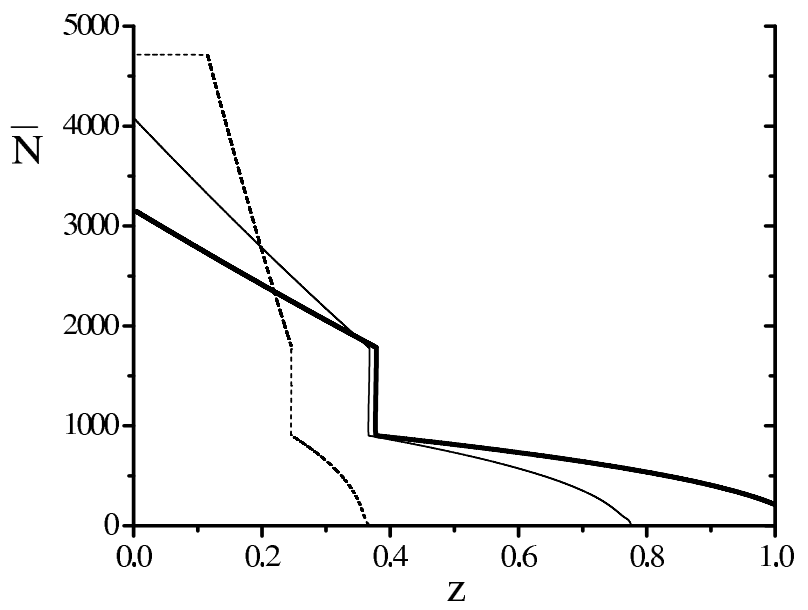

FIG. 9: Average aggregation number, $\bar{N}(z)$, as a function of height, $z$, in the phase coexistence region for three different parameters associated with the gravitational field, $\beta=0.6$ (thick solid curve), $\beta=1.0$ (thin solid curve), $\beta=3.5$ (dash curve). The jump corresponds to the position of the phase boundary, $x$, the nematic phase is at the bottom, $0<z<$ $x$, and the isotropic phase is on the top, $x<z<1$. The parameters used: $p=40, \delta=15, \Phi=0.255$.

phase. Instead, we will consider the average concentration in the nematic phase, $\Phi_{n}$, which is assumed to be at the bottom, $z<x$, and the average concentration in the isotropic phase, $\Phi_{i}$, at the top $z>x$. where $x$ is the height of the phase boundary:

$$
\begin{aligned}
\Phi_{n} & =\frac{1}{x} \int_{0}^{x} \phi_{n}(z) d z \\
\Phi_{i} & =\frac{1}{1-x} \int_{x}^{1} \phi_{i}(z) d z
\end{aligned}
$$

The concentrations are related via the conservation of mass condition $\Phi=x \Phi_{n}+(1-x) \Phi_{i}$, where $\Phi$ is the total subunit concentration in both phases. Note, that $\Phi_{n}$ and $\Phi_{i}$ are not fixed, they are determined from the equilibrium conditions.

The equilibrium corresponds to the equality of osmotic pressures and chemical potentials at the boundary, $z=x$. Using the expressions for the isotropic phase (9), (10) and the nematic phase, (26), (27), we can write

$$
\begin{aligned}
-\frac{1}{\sqrt{\phi_{i}(x) e^{\delta}}+\frac{\pi}{2} \phi_{i}(x)=} & \frac{\alpha(x)}{4 p}-\frac{1}{\sqrt{\frac{1}{4} \alpha(x) \phi_{n}(x) e^{\delta}}}+ \\
\frac{\phi_{i}(x)}{\sqrt{\phi_{i}(x) e^{\delta}}}+\frac{\pi}{4} \phi_{i}^{2}(x)= & \frac{\phi_{n}(x) \frac{4}{\sqrt{\pi \alpha(x)}}}{\sqrt{\frac{1}{4} \alpha(x) \phi_{n}(x) e^{\delta}}}+ \\
& \frac{\pi}{4} \phi_{n}^{2}(x) \frac{4}{\sqrt{\pi \alpha(x)}}
\end{aligned}
$$

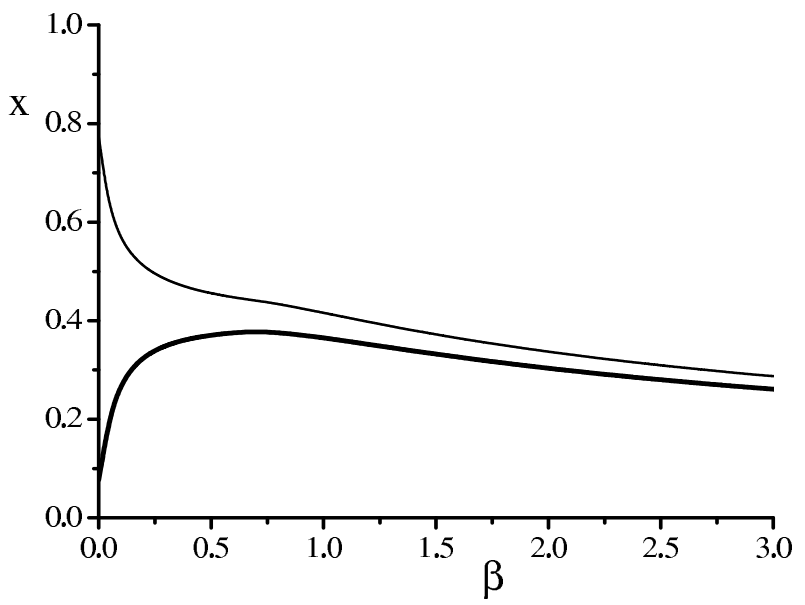

FIG. 10: The position of the boundary between two phases, $x$, as a function of $\beta=m g h / k T$ for two different total subunit concentrations: $\Phi=0.255$ (thick line) and $\Phi=0.3$ (thin line). The parameters used: $\delta=15, p=40$.

where $\alpha(z)$ is determined from eq. 25. The solution of four equations (31)-(34) along with the conservation of mass condition, $\Phi=x \Phi_{n}+(1-x) \Phi_{i}$, gives the equilibrium distribution of aggregates between two phases. Note, that the last two equations determine the boundary concentrations of the coexisting phases, $\phi_{i}(x)$ and $\phi_{n}(x)$. The form of the equations coincides with that in the absence of gravity 33 since the aggregates at the boundary have all the same potential energy and the gravitational field does not influence their behavior. Chemical potentials in both phases do not depend on the height. They can be regarded as equations for the definition of local concentrations $\phi_{i, n}(z)$ as implicit functions of height. To get the average concentrations of coexisting phases, $\Phi_{n}$ and $\Phi_{i}$, as well as the boundary position, $x$, we have to integrate the local concentrations. However, it only leads to implicit definition of local concentrations. This results in a system of integral equations which can be hardly solved. See Appendix how to avoid this difficulty.

In contrast to the phase separation in a homogeneous system, the coexisting phases in a gravitational field exhibit the concentration gradient and so, the average concentrations of the phases, $\Phi_{i}$ and $\Phi_{n}$, do not correspond to the onset concentrations of the phase transition. This is because they both depend on the total concentration, $\Phi$. In order to find the values of the total concentration signifying the phase separation boundaries, we put in the equations $x=0$ for the onset of the isotropic phase and $x=1$ for the onset of the nematic phase. Solving the above equations with respect to $\Phi$, the boundaries of the coexistence region can be obtained. Fig. 7 represents the typical phase diagram in the variables $\beta-\Phi$. The two curves diverges, thus, the gravitational field significantly broaden the coexistence region. Starting at some point in the isotropic phase at zero field and moving vertically on the plot, i.e. increasing the field, at some $\beta$ we enter 


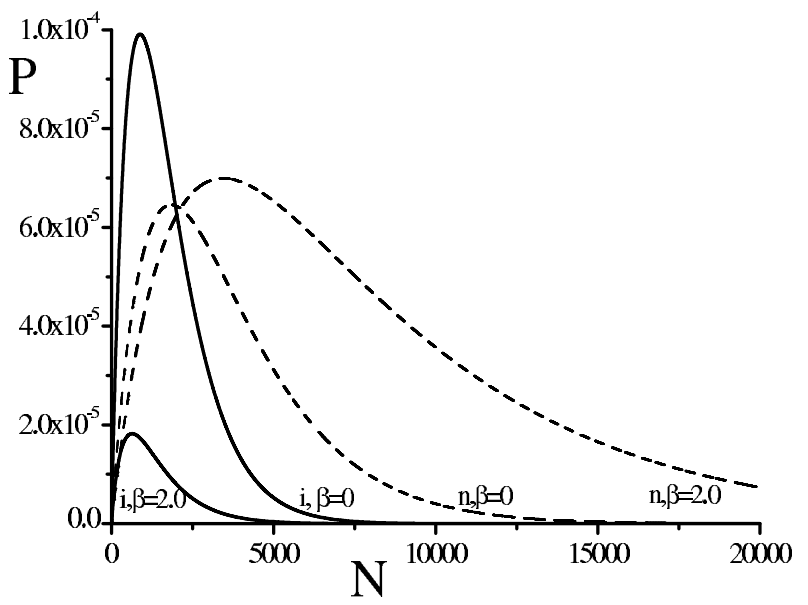

FIG. 11: Length distribution functions in the nematic phase, $P_{n}(N)$ (dash curves), and in the isotropic phase, $P_{i}(N)$ (solid curves), for two cases: in the absence of the field, subscript $\beta=0$, and in the field, subscript $\beta=2.0$. The parameters used: $p=40, \delta=15, \Phi=0.255$.

the phase separation region: increasing local concentration at the bottom reaches the limiting value and the nematic phase appear at the bottom. Increasing gravity in the nematic phase leads to the rarefaction on the top, what gives rise to the isotropic phase. Thus, the gravity facilitates the isotropic to nematic phase transition.

Since we describe the self-assemble system, which is sensitive to the variational of external conditions, in particular to the change of the temperature, it is useful to plot the phase diagram in the variables $\delta-\Phi$, where the end energy, $\delta$, usually has the inverse temperature dependence. The result is presented in Fig. 8. Here the inner phase diagram (dark pattern) is the coexistence region in the absence of the field in accordance with refs ${ }^{25.34}$ As we see, to induce the phase separation, $\delta$ should exceed some critical value, for small $\delta$ the aggregates are too short and the nematic phase never appears. (Compare it with theoretically predicted and experimental phase diagrams. $)^{25.40}$ The phase diagram depends strongly on the stiffness parameter $p$. In the limit of rigid rods, $p \rightarrow \infty$, the concentrations of coexisting phases tend to Y-axis, while for flexible aggregates, there is a little gap, i.e. stiff aggregates much easily form the nematic phase than the flexible ones. The gravitational field severely increases the coexistence region (light pattern) in both cases. So, for strong enough gravitational fields the left border can approach the Y-axis, signifying the absence of the pure isotropic phase for high values of $\delta$ even for very low concentrations. This situation should be taken into account, when the self-assembled aggregates are subject to the ultracentrifugal field.

Let us focus on the coexistence region in more details. The solution of four equations (31)-(34) along with the conservation of mass condition for each $\beta$ gives not only the average concentrations in the coexisting phases, it

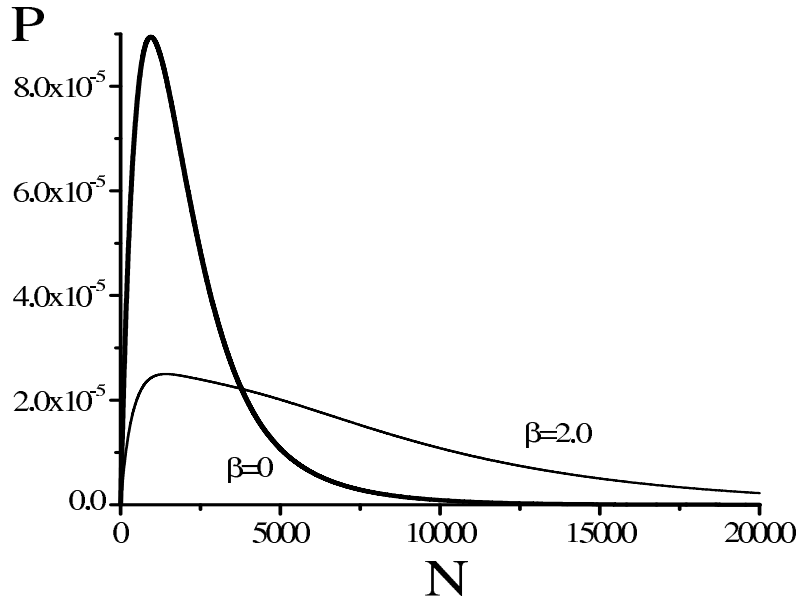

FIG. 12: The sum of the length distribution functions of the nematic and isotropic phases, presented in Fig. 11, $P(N)=$ $x P_{n}(N)+(1-x) P_{i}(N)$ for $\beta=0$ (thick line) and $\beta=2.0$ (thin line). The parameters used: $p=40, \delta=15, \Phi=0.255$.

also gives the position of the phase boundary, $x$, and the concentrations at the boundary, $\phi_{i}(x)$ and $\phi_{n}(x)$. This information is enough to obtain the full concentration profile composed of two parts, $\phi_{n}(z)$ at $0<z<x$ and $\phi_{i}(z)$ at $x<z<1$. This profiles can be converted into the average aggregation numbers in both phases:

$$
\begin{aligned}
\bar{N}_{i}\left(\phi_{i}(z)\right) & =\sqrt{\phi_{i}(z) e^{\delta}} \\
\bar{N}_{n}\left(\phi_{n}(z)\right) & =\sqrt{(2 \sqrt{\pi} p)^{2 / 3} \phi_{n}^{5 / 3}(z) e^{\delta} / 4}
\end{aligned}
$$

The resulting plot for different $\beta$ is shown in Fig. 9 (Actually, we plot $z_{i, n}(\phi)$ and thus, $z_{i, n}(N)$ as explained in Appendix). The average aggregation number in the isotropic phase exhibits the square root behavior on height, and it has almost linear dependence in the nematic phase in accordance to our previous estimates. The only exception is the region at the very bottom for high gravitational field $\beta=3.5$, where $\bar{N}_{n}$ does not depend on $z$. This is because the concentration at the bottom for high gravitational fields is very high and the second virial approximation does not work. Thus, after correcting this approximation, the sharp edge on the dashed curve should smooth out. The length of the concentration jump at the phase boundary does not depend on the field, since the field can only shift the phase boundary and it does not influence the equilibrium of the aggregates lying in the same height.

As in the case of monodisperse rods of fixed length, the gravity can either increase or decrease the volume of the nematic phase depending on the total subunit concentration (Fig. 10). If the total concentration is close to the isotropic phase boundary and the volume of the nematic phase is low, the gravitational field induces the increase of the nematic phase, so the volume increases until exhausting of the isotropic phase. Since then, the 
volume gradually decreases indicating the sinking of the aggregates in the nematic phase (solid line). If the total concentration is close to the nematic phase boundary, the aggregates in the nematic phase can only sink, decreasing the volume (thin line).

Now, let us address the question of the polydispersity of the nematic and the isotropic phases in the gravitational field. The length distribution functions of aggregates in coexisting phases, $P_{i}(N)$ and $P_{n}(N)$ can be calculated as

$$
\begin{aligned}
P_{i}(N) & =\frac{N}{1-x} \int_{x}^{1} \frac{\phi_{i}(z)}{\bar{N}_{i}^{2}(z)} \exp \left(-\frac{N}{\bar{N}_{i}(z)}\right) d z \\
P_{n}(N) & =\frac{N}{x} \int_{0}^{x} \frac{\phi_{n}(z)}{\bar{N}_{n}^{2}(z)} \exp \left(-\frac{N}{\bar{N}_{n}(z)}\right) d z
\end{aligned}
$$

The result is presented in Fig. 11. See Appendix and especially eqs. A.7, A.8 for the details of the calculation. The gravity induces the transition of subunits from the isotropic to the nematic phase. So, the area of the isotropic distribution function, $P_{i}(N)$, decreases with increasing gravity, while the area of $P_{n}(N)$ increases with $\beta$. Nevertheless, the distribution of lengths in the nematic phase is much broader in the presence of the field, i.e. the gravity increases seriously the polydispersity of the nematic phase. This is for account of the fraction of extremely long aggregates induced by the gravity, while the fraction of short aggregates remains almost unchanged. The isotropic phase looses foremost long, and thus, heavy aggregates, but the change in its polydispersity is not so drastic as in the nematic phase. Hence, the precipitation into nematic phase is more favorable process than the growth in the isotropic phase. To describe the polydispersity of the whole two phase system, we can consider the sum of the length distribution functions, $P=x P_{n}(N)+(1-x) P_{i}(N)$. The area of this function is proportional to the total number of subunits in the system, so its value is the same for any $\beta$. The plot of $P(N)$ is shown in the Fig. 12. It brings us to the conclusion that the system as a whole is more polydisperse in the gravitational field. Sharply picked function in the absence of gravity fade out with increasing gravitational field. Note, however, that the maximum of the curve almost does not change. This is the consequence of the redistribution of subunits between two phases without considerable growth of the aggregates.

\section{CONCLUSION}

Although the gravitational field is often neglected in the description of physicochemical processes, it may have noticeable influence on such biological heavy objects as microtubules or actin filaments, essential components of living cells. The equilibrium properties of such objects is influenced even by the earth gravitational acceleration. The fields in an ultracentrifuge can multiple the effect of gravity.
In this paper we focus on the major effects of the gravitational field on the process of self-assembly of linear aggregates. It is shown, that the concentration gradient induced by the gravitational field leads to the redistribution of aggregates of different lengths (and thus, masses) on different heights: the local concentration of the self-assembled rods interacting via the second virial coefficient decreases linearly with height in contrast to barometric distribution of aggregates with fixed length. This prediction can be directly checked experimentally in the rotating clinostat experiments. In turn, the average length of the aggregates has a square root dependence in contrast to hyperbolic dependence found for ideal aggregates. ${ }^{19}$ We also show, that except the redistribution of aggregates with height, the gravity induces the overall growth of the aggregates. The stiffness of the aggregates enhance the growth under gravity. Strong enough gravitational field can provoke the isotropic to nematic phase transition, which is reflected in the significant broadness of the phase coexistence region. The transition manifests itself in the precipitation of the aggregates from the isotropic to the nematic phase regardless their length. Furthermore, the length distribution of the aggregates is found to be very sensitive to the gravitational field: the gravity induces considerable polydispersity in the nematic phase. Taken together, the effect of gravity can lead to a series of experimentally relevant observations which are interesting from fundamental point of view. 


\section{APPENDIX: ,}

Equations $\mu_{i, n}=$ const (9), (26) do not allow to obtain the analytical expression of local concentrations $\phi_{i, n}(z)$ as a function of $z$ except for two asymptotes eqs. (111), (16). To this end, the total concentration, $\Phi=\int \phi(z) d z$, and the average lengths, $\bar{N}_{i, n}(z)$, are, in fact, the integral equations for the functions $\phi_{i, n}(z)$, the functional forms of which are determined from the chemical potentials. In general, it is rather difficult to solve such system of equations. However, we can employ the structure of equations (9), (26), which allows to get analytically the inverse functions, $z_{i, n}(\phi)$. Namely, for the isotropic phase

$$
z_{i}(\phi)=x-\frac{1}{\beta}\left[\frac{1}{\sqrt{\phi_{i}(x) e^{\delta}}}-\frac{1}{\sqrt{\phi e^{\delta}}}-\frac{\pi}{2}\left(\phi_{i}(x)-\phi\right)\right]
$$

Here we assume the isotropic phase is situated above the phase boundary, $x<z_{i}<1$, and the boundary concentration is $\phi_{i}(x)$. In case of the homogeneous phase, one can put in this expression $x=0$. Analogously, the inverse function for the nematic phase yields in the form

$$
\begin{aligned}
z_{n}(\phi)= & x+\frac{5 \pi^{1 / 2} e^{\delta / 2}\left(\phi_{n}^{2 / 3}(x)-\phi^{2 / 3}\right)}{2^{4 / 3} \pi^{1 / 6} p^{1 / 3} e^{\delta / 2} \beta}+ \\
& \frac{4\left(1 / \phi^{5 / 6}-1 / \phi_{n}^{5 / 6}(x)\right)}{2^{4 / 3} \pi^{1 / 6} p^{1 / 3} e^{\delta / 2} \beta}
\end{aligned}
$$

The two expressions should be accompanied by the boundary conditions, $z_{i}\left(\phi=\phi_{i}(1)\right)=1$ and $z_{n}(\phi=$ $\left.\phi_{n}(0)\right)=0$.

With this, the average concentrations in the phases are

$$
\begin{aligned}
\Phi_{i} & =\frac{1}{1-x} \int_{\phi_{i}(x)}^{\phi_{i}(1)} \phi \frac{\partial z_{i}(\phi)}{\partial \phi} d \phi \\
\Phi_{n} & =\frac{1}{x} \int_{\phi_{n}(0)}^{\phi_{n}(x)} \phi \frac{\partial z_{n}(\phi)}{\partial \phi} d \phi
\end{aligned}
$$

where the value of the local concentration is $\phi_{n}(0)$ at the bottom and $\phi_{i}(1)$ on the top.

The derivatives in the integrals are

$$
\begin{aligned}
\frac{\partial z_{i}(\phi)}{\partial \phi} & =-\frac{\pi}{2 \beta}\left(1+\frac{1}{\pi \phi \sqrt{\phi e^{\delta}}}\right) \\
\frac{\partial z_{n}(\phi)}{\partial \phi} & =-\frac{5}{3} \frac{1}{(2 \sqrt{\pi} p)^{1 / 3} \phi^{11 / 6} \beta}\left(\sqrt{\pi} \phi^{3 / 2}+e^{-\delta /(2 \mathrm{~A}) .6)}\right.
\end{aligned}
$$

Thus, the average concentrations can be integrated analytically.

The global length average of the aggregates in both phases, (13) and (30), can be written as integrals on $\phi$ :

$$
\begin{aligned}
\left\langle N_{i}\right\rangle & =\Phi_{i} e^{\delta / 2} / \int_{\phi_{i}(x)}^{\phi_{i}(1)} \phi^{1 / 2} \frac{\partial z_{i}(\phi)}{\partial \phi} d \phi \\
\left\langle N_{n}\right\rangle & =\frac{\Phi_{n} e^{\delta / 2}}{(2 \sqrt{\pi} p)^{1 / 3}} / \int_{\phi_{n}(0)}^{\phi_{n}(x)} \phi^{5 / 6} \frac{\partial z_{n}(\phi)}{\partial \phi} d \phi(A .8
\end{aligned}
$$

The length distribution functions (37), (38) yield in the form

$$
\begin{aligned}
P_{i}(N)= & \left.\frac{N}{1-x} e^{-\delta} \int_{\phi_{i}(x)}^{\phi_{i}(1)} \exp \left(-\frac{N}{\sqrt{\phi e^{\delta}}}\right) \frac{\partial z_{i}(\phi)}{\partial \phi}(\phi \phi)\right) \\
P_{n}(N)= & \frac{N}{x} \frac{4 e^{-\delta}}{(2 \sqrt{\pi} p)^{2 / 3}} \int_{\phi_{n}(0)}^{\phi_{n}(x)} \phi^{-2 / 3} \times \\
& \left.\exp \left(-\frac{2 N}{\sqrt{(2 \sqrt{\pi} p)^{2 / 3} \phi^{5 / 3} e^{\delta}}}\right) \frac{\partial z_{n}(\phi)}{\partial \phi}(1) .10\right)
\end{aligned}
$$

where we used (35), (36) for the averages in the isotropic and the nematic phases.

Solution of algebraic eqs. (33), (34), (A.3), (A.4) along with the conservation mass condition, $\Phi=x \Phi_{n}+(1-$ $x) \Phi_{i}$, gives the phase diagram as well as the equilibrium average concentrations in the coexisting phases.
1 Cates M. E., Candau S. J., J. Phys.: Condens. Matter, 2, 6869 (1990).

${ }^{2}$ Greer S., in Advances in Chem. Phys., XCIV, Polymeric Systems, edited by Prigogine I. and Rice S., (Interscience, N.Y. 1996).

3 Penrose, R. Shadows of the Mind (Oxford University Press, N.Y. 1994).

${ }^{4}$ Dogterom M. in Physics of Bio-molecules and Cells, edited by Flyvbjerg H, Julicher F., Ormos P., David F., Les Houches Session LXXV (EDP Sciences, Springer-Verlag, 2002).

${ }^{5}$ Dogterom M., Yurke B., Science, 278, 856 (1997).
${ }^{6}$ Scholey J. M., Rogers G. C., Sharp D.J., The Journal of Cell Biology, 154(2), 261 (2001).

7 Panda D., Chakrabarti G., Hudson J., Pigg K., Miller H., Wilson L., Himes R., Biochemistry, 39, 5075 (2000).

8 Derry W., Wilson L., Khan I., Luduena R., Jordan M. A., Biochemistry, 36, 3554 (1997).

9 Anacker E., Rush R., Johnson J., J. Phys. Chem., 68 (1), 81 (1964).

10 Shah C., Zhi-Qi Xu C., Vickers J., Williams R., Biochemistry, 40, 4844 (2001).

${ }^{11}$ Hlavanda E., Kovacs J., Olah J., Orosz F., Medzihradszky K., Ovadi J., Biochemistry, 41, 8657 (2002). 
12 Tabony J., Glade N., Papaseit C., Demongeot J., Cell Biology and Biotechnology in Space, 19 (2002) and Glade N., Tabony J., J. Phys. IV France, 11, Pr6-255 (2001).

13 Tabony J., Glade N., Demongeot J., Papaseit C., Langmuir, 18, 7196 (2002).

14 Tabony J., Glade N., Papaseit C., Demongeot J., J. Phys. IV France 11, Pr6-239 (2001).

15 Tabony J., Pochon N., Papaseit C., Advances in Space Research, 28(4), 529 (2001).

16 Claassen D., Spooner B., Advances in Space Research, 17(6-7), 151 (1995).

17 Dag O., Ahari H., Coombs N., Jiang T., ArocaOuellette P., Petrov S., Sokolov I., Verma A., Vovk G., Young D., Ozin G., Reber C., Pelletier Y., Bedard R., Advanced Materials, 9(15), 1133 (1997).

18 Hong J., J. Crystal Growth, 181, 459 (1997) and references therein.

19 Duyndam A., Odijk T., Langmuir, 9, 1160 (1993).

20 Duyndam A., Odijk T., Langmuir, 14, 2577 (1998).

21 Dogic Z., Philipse A., Fraden S., Dhont J., J. Chem. Phys., 113(18), 8368 (2000).

22 Baulin V.A., Khokhlov A.R., Phys. Rev. E, 60(3), 2973 (1999).

23 Safran, S. A. Statistical Thermodynamics of Surfaces, Interfaces and Membranes (Addison-Wesley, Reading, MA, 1994).

24 Odijk T., Curr. Opin. in Col. \& Int. Sci., 1, 337 (1996).

25 Taylor M., Herzfeld J., J. Phys.: Condens. Matter, 5, 2651 (1993).

${ }^{26}$ Gelbart W., Ben-Shaul A., McMullen W., Masters A., J.
Phys. Chem., 88, 861 (1984).

27 Gelbart W., McMullen W., Ben-Shaul A., J. Physique, 46, 1137 (1985).

28 McMullen W., Gelbart W., Ben-Shaul A., J. Chem. Phys., 82(12), 5616 (1985).

${ }^{29}$ Cuesta J., Sear R., Eur. Phys. J. B, 8, 233 (1999).

30 van der Schoot P., Cates M., Langmuir, 10, 670 (1994).

31 van der Schoot P., Europhys. Lett., 39(1), 25 (1997).

32 van der Schoot P., J. Phys. II (France), 5, 243 (1995).

33 van der Schoot P., Cates M., Europys. Lett., 25 (7), 515 (1994).

34 van der Schoot P., Macromolecules, 27, 6473 (1994).

35 Odijk T., Macromolecules, 19, 2313 (1986).

36 Onsager L., Ann. (N.Y.) Acad. Sci., 51, 627 (1949).

37 In the isotropic phase the integral in the exponent of eq. (4) can be calculated directly. It yields in the form $2 / v \int C(N, \Omega, z) B_{2}(\gamma) d N d \Omega=\pi / 2 \phi N$. Here the equality $\int|\sin \gamma| d \Omega=\pi^{2}$ was used.

38 Integration of (11) on the whole range of $z$ allows to relate $\bar{N}_{i, 0}$ and $\Phi_{i}$. With this, the substitution of (12) into eq. (13) and subsequent integration gives (14).

39 To calculate $z_{c r}$ we assume that the subunits concentration for $z>z_{c r}$ is negligibly small and $\Phi_{i}=\int_{0}^{z_{c r}} \phi_{i}(z) d z$. Integration and solution of this equation with respect to $\bar{N}_{i, 0}$ gives $\bar{N}_{i, 0}=\sqrt{e^{\delta}\left(\Phi_{i}+z_{c r}^{2} \beta / \pi\right) / z_{c r}}$. Substitution of this result into (17) and solution of the subsequent equation $\bar{N}_{i}(z)=0$ gives the crossover height $z_{c r}=\sqrt{\Phi_{i} \pi / \beta}$.

${ }^{40}$ Herzfeld J., Acc. Chem. Res., 29, 31 (1996). 